\title{
Influência dos fatores ambientais sobre a distribuição da comunidade de invertebrados bentônicos em canais de uma planície de inundação neotropical
}

\author{
Danielle Katharine Petsch ${ }^{{ }^{*}}$ \\ Gisele Daiane Pinha ${ }^{1}$ \\ Flávio Henrique Ragonha ${ }^{1}$ \\ Alice MichiyoTakeda ${ }^{2}$
${ }^{1}$ PPG em Ecologia de Ambientes Aquáticos Continentais, Universidade Estadual de Maringá Avenida Colombo, 5790 CEP 87020-900 Maringá - PR
${ }^{2}$ Universidade Estadual de Maringá/DBI/NUPELIA/PEA *Autor para correspondência
dannypetsch@hotmail.com

Submetido em 09/11/2012

Aceito para publicação em 07/05/2013

\section{Resumo}

Um dos temas centrais em ecologia é a importância relativa dos processos locais e regionais na determinação da estrutura de comunidades, pois ambos os processos podem agir como filtros na composição das comunidades locais. Nesse sentido, este estudo analisou a influência dos fatores ambientais sobre a distribuição da comunidade de invertebrados bentônicos em diferentes canais da planície de inundação do alto rio Paraná, por meio de coletas trimestrais realizadas de março a dezembro de 2010. A partir de dados bióticos e abióticos foi realizada uma Análise de Correspondência Canônica, onde foi possível agrupar os centros dos rios Ivinhema e Paraná e do canal Ipoitã em relação aos demais pontos, principalmente pelos maiores valores de profundidade e velocidade e pelos táxons típicos de ambientes lóticos, como Harpacticoida, Haplotaxidae e Narapidae, e o centro do canal Curutuba, com L. fortunei. Pode-se concluir que a velocidade de fluxo, a textura granulométrica e a matéria orgânica do sedimento foram fatores estruturadores da comunidade bentônica, determinando a distribuição dos invertebrados tanto entre os diferentes canais como entre as regiões marginais e centrais desses ambientes, proporcionando maior ou menor disponibilidade de recursos e heterogeneidade ambiental.

Palavras-chave: Ambientes lóticos; Textura granulométrica; Variação espacial

\section{Abstract}

Influence of environmental factors on the benthic invertebrates community distribution in channels of a neotropical floodplain. One of the central themes in ecology is the relative importance of local and regional processes for determining the communities' structure, since both processes may behave as filters in the composition of local communities. Thus, this study analyzed the influence of environmental factors on the benthic invertebrate community distribution in different channels of the Upper Paraná River floodplain, through quarterly samplings conducted from March to December 2010. Through the biotic and abiotic data, we performed a Canonical Correspondence Analysis, where it was possible to visualize the centers of Ivinhema and Paraná 
rivers and Ipoitã channel separate from other points by high values of depth and velocity and taxa typical of lotic environments, such as Harpacticoida, Haplotaxidae, and Narapidae, and the center of the Curutuba channel, with $L$. fortunei. One may conclude that flow velocity, granulometric texture, and sediment organic matter were structuring factors of the benthic community, determining the distribution of invertebrates both among the various channels and between the marginal and central regions in these environments, providing greater or lesser availability of resources and environmental heterogeneity.

Key words: Lotic environments; Granulometric texture; Spatial variation

\section{Introdução}

Um dos objetivos nos estudos ecológicos tem sido explicar os padrões das assembleias biológicas através de um conjunto de áreas que são espacialmente distintos, mas sujeitos ao mesmo pool regional de espécies (MOORE; SWIHART, 2007). Neste contexto, um dos temas centrais é a importância relativa dos processos locais e regionais na determinação da estrutura de comunidades (RICKLEFS, 1987), pois ambos os processos podem agir como filtros na composição das mesmas (POFF, 1997).

A planície de inundação do alto rio Paraná é uma área de grande importância ecológica, pois agrega um mosaico de habitats aquáticos, terrestres e de transição, onde diferenças físicas e químicas criam uma alta heterogeneidade ambiental capaz de comportar uma elevada diversidade biológica (THOMAZ et al., 2007). Nesse sentido, o canal principal e os canais secundários têm um papel fundamental em uma planície de inundação, pois são considerados ambientes de comunicação entre diversos ecótopos (PAILLEX et al., 2007).

Dentre as comunidades aquáticas, a de invertebrados bentônicos pode ser considerada como um dos componentes desses ecossistemas que apresentam ampla distribuição, além de ser fortemente influenciada pelas condições ambientais e pela sua pouca mobilidade (MORETTI; CALLISTO, 2005; WÜRDIG et al., 2007), características interessantes que permitem explorar as relações entre a estrutura de comunidades e fatores ambientais. A influência das variáveis limnológicas, como: velocidade de fluxo, nível fluviométrico (TAKEDA et al., 2004), composição do sedimento (PRÍNCIPE; CORIGLIANO, 2006) e teor de matéria orgânica (TAKEDA et al., 2004) sobre esta comunidade conferem características próprias a cada ambiente, influenciando diretamente a elevada biodiversidade em planícies de inundação (SÚAREZ et al., 2001) e interferindo na presença e distribuição espacial dos organismos (BECHARA, 1996; TAKEDA; FUJITA, 2004).

Áreas naturais preservadas mantêm a integridade de um número considerável de ecossistemas aquáticos, o que as torna de grande importância para a diversidade ecológica e à manutenção dos padrões estruturais destes ambientes (AGOSTINHO et al., 2005). Nesse aspecto, estudos com estas perspectivas ecológicas têm sido realizados para a melhor compreensão das interações dos organismos e funcionamento destes ecossistemas (CALLISTO et al., 2001; HENRIQUES-OLIVEIRA et al., 2003; SURIANO; FONSECA-GESSNER, 2004; SILVA et al., 2007).

Visando a melhor compreensão da estrutura da comunidade de invertebrados bentônicos, foi analisada a influência dos fatores ambientais sobre a distribuição desses organismos no canal principal e em canais secundários da planície de inundação do alto rio Paraná. Assim, foi tomado por pressuposto que as diferenças na composição, riqueza e densidade dos táxons entre os locais amostrados são uma consequência da influência dos fatores ambientais.

\section{Materiais e Métodos}

\section{Área de estudo}

Situada no trecho compreendido entre a foz do rio Paranapanema e a foz do rio Ivinhema, a bacia hidrográfica da planície de inundação do alto rio Paraná ocupa uma vasta área, que em território brasileiro supera $802.150 \mathrm{~km}^{2}$, abrangendo os estados do Paraná e Mato Grosso do Sul (SOUZA FILHO; STEVAUX, 1997). 
Sete áreas de amostragem foram estabelecidas em diferentes canais na planície de inundação do alto rio Paraná: rio Ivinhema, canal Ipoitã, rio Baía, canal Baía, canal Curutuba, rio Paraná e canal Cortado (Figura 1). O rio Ivinhema possui profundidade média de 3,9 m, apresenta vegetação diferenciada em suas margens que vai desde vegetação herbácea até a formação de extensas áreas com mata ripária em diferentes estágios de regeneração. Este rio corre paralelo ao rio Paraná em seu curso inferior (área amostrada), conectando-se com rio Baía pelo canal Curutuba e ao rio Paraná pelo canal Ipoitã e dois outros canais. O canal Ipoitã é um ambiente sinuoso que liga o rio Paraná ao rio Ivinhema, possui profundidade média de 3,2 m e suas margens são compostas de vegetação arbustiva-arbórea.

O rio Baía é considerado como ambiente entre lêntico e lótico, com largura variada e profundidade média de 3,2 m, enquanto o canal Baía se estende da confluência do rio Baía e canal Curutuba ao rio Paraná, com profundidade média de 2,0 $\mathrm{m}$ e vegetação marginal composta de campos de pastagens. Ambos apresentam uma velocidade de fluxo reduzida quando comparado aos demais canais.

O canal Curutuba, que liga o rio Baía ao rio Ivinhema, possui profundidade média de $2,7 \mathrm{~m}$ e suas margens apresentam vegetação arbustivo-arbórea, enquanto o canal Cortado é um canal secundário do rio Paraná, com profundidade média de 1,3 m. Finalmente, o rio Paraná, que apresenta largura variada e ilhas e barras ao longo de seu curso. A profundidade média é de 4,0 m, na área amostrada, mas pode atingir até $15 \mathrm{~m}$ em alguns locais (adaptado de UNIVERSIDADE ESTADUALDE MARINGÁ, 2000).

\section{Coleta e análise de dados}

As amostras foram coletadas trimestralmente, de março a dezembro de 2010, com o auxílio de um pegador

FIGURA 1: Localização dos rios e canais amostrados na planície de inundação do alto rio Paraná.

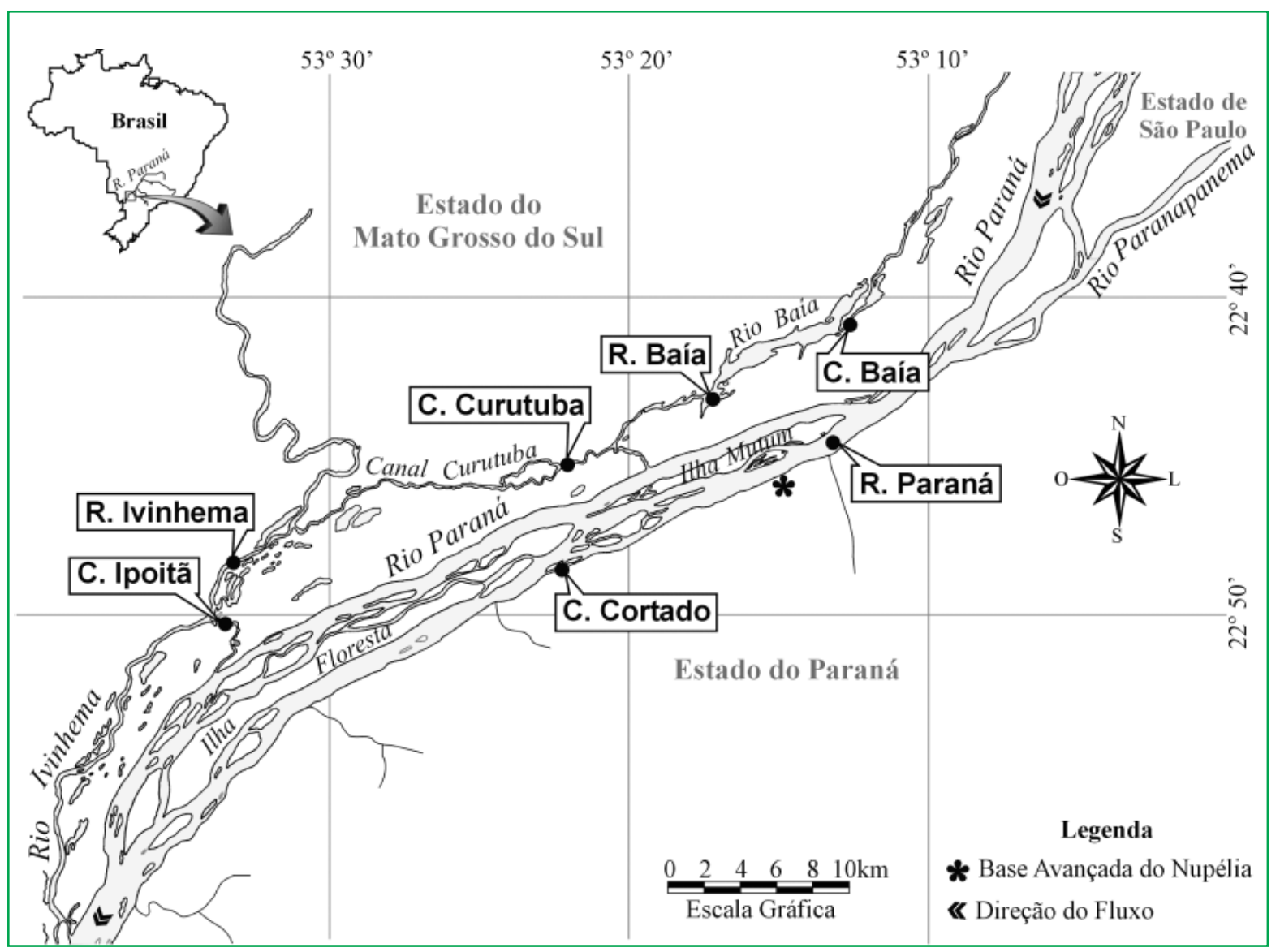


de fundo tipo Petersen modificado $\left(0,0345 \mathrm{~m}^{2}\right)$. Para cada ambiente foram determinados três pontos, em transecto: margem direita, margem esquerda e região central.

Em cada ponto de amostragem foram coletadas três amostras para o estudo biológico e uma amostra para a análise sedimentológica. Todo material coletado para a análise biológica foi acondicionado em recipientes e levado para a base avançada do Nupelia/Porto Rico-PR, onde foi realizada a lavagem em uma série de peneiras de malhas: 2,0 $\mathrm{mm} ; 1,0 \mathrm{~mm}$ e $0,2 \mathrm{~mm}$. Os invertebrados retidos nas duas primeiras malhas foram retirados e imediatamente fixados em álcool $80 \%$.

O sedimento retido na última peneira também foi fixado com álcool $80 \%$ para a posterior triagem sob microscópio estereoscópico no Laboratório de Zoobentos do Nupelia/UEM, onde os invertebrados bentônicos foram contados e identificados utilizandose as chaves de Pérez (1988) e Mugnai et al. (2010). O número total de indivíduos encontrados por amostra foi transformado em densidade (ind $/ \mathrm{m}^{2}$ ) e a partir das três amostras de cada ponto foi calculada a densidade média do ponto.

A composição granulométrica foi realizada utilizando-se a escala de Wentworth (1922). A estimativa do conteúdo de matéria orgânica do sedimento foi obtida pela queima de $20 \mathrm{~g}$ de sedimento seco em mufla a $560^{\circ} \mathrm{C}$, por cerca de quatro horas. Os dados das variáveis abióticas da água foram obtidos concomitantes às coletas de zoobentos, pela equipe do Laboratório de Limnologia Básica do Nupelia/UEM, sendo mensurados os seguintes: temperatura da água $\left({ }^{\circ} \mathrm{C}\right)$ e oxigênio dissolvido $(\mathrm{mg} / \mathrm{L})$, obtidos através de oxímetro portátil com aparelho YSI; condutividade elétrica $(\mu \mathrm{S} / \mathrm{cm})$ através de condutivímetro portátil da marca Digimed; profundidade, através do aparelho Ecosonda Hondex PS 7; pH, obtido através do potenciômetro digital portátil da marca Digimed; velocidade de fluxo $(\mathrm{m} / \mathrm{s})$, mensurada com o auxílio do correntômetro General Oceanics, model 2035 MKIV; turbidez (NTU), estimada utilizando-se um turbidímetro digital portátil marca LaMote-2008.

As amostras de água foram armazenadas em caixas térmicas e levadas para a base avançada Universidade de Maringá, onde foram filtradas através de filtros GF 52-C em membranas ( $<10$ horas após a colheita) e imediatamente congeladas $\left(-20^{\circ} \mathrm{C}\right)$ para posterior análise de dissolução nutrientes. A água foi também congelada a $-20^{\circ} \mathrm{C}$ antes da filtragem para medir as concentrações de fósforo total. As membranas foram usadas para quantificar a clorofila (extração em acetona e leitura num espectrofotómetro, de acordo com Golterman et al., 1978).

As concentrações de Nitrogênio total foram analisados com o método de persulfato com oxidações de qualquer composto nitrogenado para N-nitrato (BERGAMIN et al., 1978). Este íon foi determinado num espectrofotômetro após redução a $\mathrm{N}$-nitrito, na presença de cádmio, usando um sistema de fluxo de injeção (GINÉ et al., 1980). As concentrações de $\mathrm{N}$-amoniacal foram quantificadas pelo método do indofenol azul, lido em espectrofotômetro (MacKERETH et al., 1978). As concentrações de Fósforo total foram mensuradas em espectrofotômetro, de acordo com Golterman et al. (1978).

Como os invertebrados bentônicos estão intimamente relacionados ao sedimento (MONANHOLIMARTINS; TAKEDA, 1999; KOWNACKI et al., 2000; BUSS et al., 2004), analisou-se a textura granulométrica e o teor de matéria orgânica tanto para os diferentes ambientes como para os pontos, centrais e marginais.

Para sumarizar os dados bióticos e abióticos foi realizada uma Análise de Correspondência Canônica (CCA) (TER BRAAK, 1986; LEGENDRE; LEGENDRE, 1998), uma análise multivariada de ordenação realizada para verificar a formação de agrupamentos entre os pontos e identificar os fatores que mais influenciam os eixos. Foram utilizados os dados das variáveis abióticas da água que mais influenciam na distribuição da comunidade bentônica, textura granulométrica e teor de matéria orgânica do sedimento, além da densidade dos táxons de invertebrados.

As análises foram realizadas utilizando-se o programa PC-ORD (versão 5.01) e os gráficos confeccionados no programa Statistica (versão 7.0). 


\section{Resultados e Discussão}

Em relação às variáveis limnológicas dos ambientes no período amostrado, pode-se observar que o rio Paraná foi caracterizado pelos maiores valores de oxigênio dissolvido, $\mathrm{pH}$ e alcalinidade e pelas menores concentrações de clorofila e fósforo e menor turbidez da água (Tabela 1). Os demais ambientes caracterizaramse por valores intermediários dessas variáveis, com destaque para os altos valores de clorofila, nitrogênio e fósforo no rio Baía, juntamente com os menores valores de velocidade da água.

Em relação à textura granulométrica de cada ambiente foram registrados maiores valores de lama e matéria orgânica no rio Baía e canal Baía, condições características de ambientes lênticos, enquanto os menores valores foram registrados no rio Paraná, canal Ipoitã e rio Ivinhema (Figura 2). No canal Curutuba foi registrada maior porcentagem de seixos e a terceira maior porcentagem de matéria orgânica entre os ambientes.

Verificou-se que as margens possuem maior teor de matéria orgânica do que a região central (Figura 2). Em relação à textura granulométrica, foram registrados maiores valores de lama e areia fina e muito fina nas margens, enquanto no centro os maiores valores foram de areia fina e areia média. De maneira geral, maiores porcentagens de grânulos e seixos foram encontrados na região central. Em ambientes lóticos, a maior velocidade de fluxo do centro carrega os materiais sedimentares mais finos que são depositados nas margens, resultando nas maiores proporções de areia mais fina nessas áreas. Como consequência, apenas sedimentos mais grosseiros, como seixos e grânulos, permanecem nas regiões centrais, explicando os resultados observados.

Um total de 17.039 invertebrados bentônicos foi registrado, distribuídos em 27 táxons distintos (Tabela 2). Arthropoda foi o filo mais representativo, sobressaindo-se Insecta, fato esse também constatado por outros autores (LAKE, 1990; MARQUES et al., 1999; RIBEIRO; UIEDA, 2005). Muitos táxons foram comuns a todos os ambientes amostrados, como Nematoda, Bivalvia, Naidinae, Pristininae, Tubificinae, Acari, Ostracoda, Ephemeroptera, Chironomidae e Ceratopogonidae, o que demonstra a alta plasticidade ambiental desses grupos capazes de se dispersar e estabelecer em diferentes locais. Turbellaria, Haplotaxidae, Harpacticoida, Amphipoda, Lepidoptera e Chaoboridae foram menos frequentes, demonstrando ocorrência mais restrita para apenas alguns ambientes.

TABELA 1: Caracterização limnológica dos rios e canais amostrados na planície de inundação do alto rio Paraná em 2010.

\begin{tabular}{lccccccc}
\hline & R. Baía & C. Baía & C. Cortado & C. Curutuba & C. Ipoitã & R. Ivinhema & R. Paraná \\
\hline Temperatura $\left({ }^{\circ} \mathrm{C}\right)$ & 25,40 & 23,98 & 24,33 & 25,05 & 24,45 & 24,83 & 24,48 \\
Oxigênio dissolvido $(\mathrm{mg} / \mathrm{L})$ & 5,82 & 4,83 & 7,35 & 6,44 & 5,80 & 6,01 & 7,75 \\
$\mathrm{pH}$ & 6,49 & 6,14 & 7,30 & 6,61 & 6,99 & 6,82 & 7,49 \\
Condutividade elétrica $(\mu \mathrm{S} / \mathrm{cm})$ & 27,48 & 23,32 & 62,23 & 35,85 & 49,18 & 41,78 & 59,83 \\
Secchi $(\mathrm{m})$ & 1,13 & 1,46 & 1,23 & 1,19 & 1,98 & 1,06 & 4,54 \\
Turbidez $(\mathrm{NTU})$ & 9,32 & 7,26 & 11,86 & 8,82 & 6,40 & 17,18 & 3,34 \\
Alcalinidade $(\mathrm{mEq} / \mathrm{L})$ & 141,23 & 98,67 & 441,23 & 191,09 & 349,70 & 300,38 & 405,50 \\
Profundidade média $(\mathrm{m})$ & 1,93 & 1,73 & 2,02 & 2,24 & 3,89 & 4,26 & 3,17 \\
Velocidade de fluxo $(\mathrm{m} / \mathrm{s})$ & 0,01 & 0,03 & 0,06 & 0,09 & 0,08 & 0,23 & 0,17 \\
Clorofila $(\mu \mathrm{g} / \mathrm{L})$ & 6,32 & 5,05 & 2,61 & 3,27 & 2,12 & 3,13 & 0,76 \\
Nitrogênio total $(\mu \mathrm{g} / \mathrm{L})$ & 858,09 & 702,96 & 886,46 & 832,97 & 550,75 & 555,75 & 711,84 \\
Fósforo total $(\mu \mathrm{g} / \mathrm{L})$ & 41,55 & 35,54 & 22,43 & 35,14 & 20,04 & 40,15 & 9,70 \\
\hline
\end{tabular}


FIGURA 2: Textura granulométrica (A) e teor de matéria orgânica (B) nas diferentes áreas amostradas da planície de inundação do alto rio Paraná. Textura granulométrica $(\mathrm{C})$ e teor de matéria orgânica $(\mathrm{D})$ nas diferentes regiões amostradas dos canais. $\mathbf{A M F}=$ areia muito fina, $\mathbf{A F}=$ areia fina, $\mathbf{A M}=$ areia média, $\mathbf{A G}=$ areia grossa, $\mathbf{A M G}=$ areia muito grossa, $\mathbf{M D}=$ margem direita, $\mathbf{M E}=$ margem esquerda.

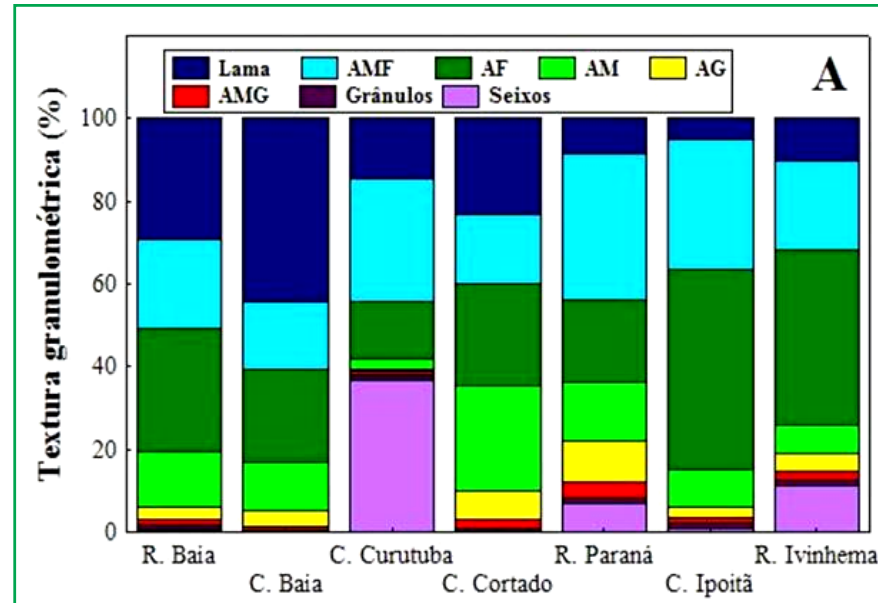

Ambientes

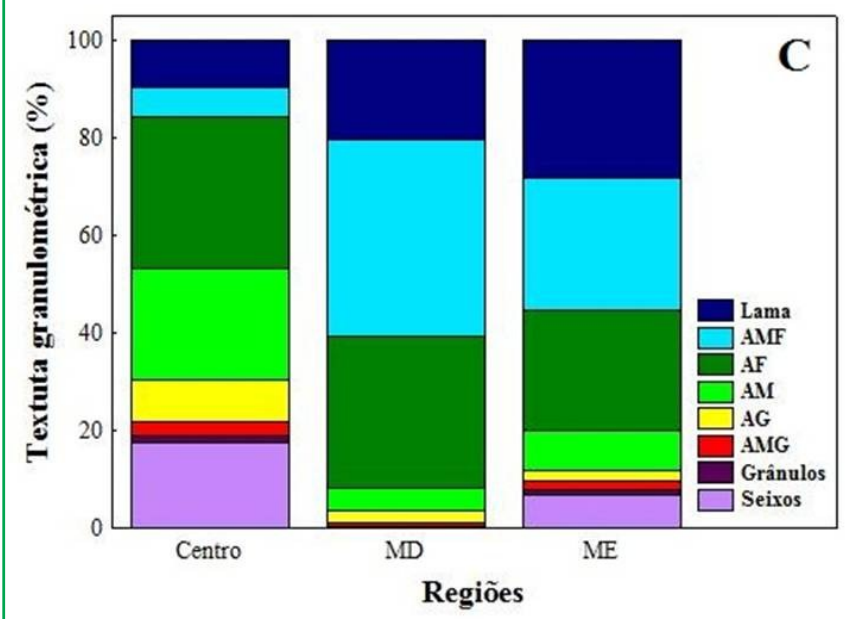

Os maiores valores de riqueza de táxons e densidade média foram registrados nos rios Ivinhema (25 táxons) e Paraná (22 táxons), provavelmente devido às condições de maior oxigenação e margens com vegetação arbórea (Figura 3). Essas características, segundo Henriques-Oliveira et al. (1999), Buckup et al. (2007) e Rosin et al. (2010), constituem como propícias para maior estabelecimento de invertebrados bentônicos. As menores riquezas foram observadas no rio Baía (16 táxons) e canal Baía (18 táxons), o que pode ser explicado pelas maiores porcentagens de matéria orgânica que reduzem o $\mathrm{pH}$ e as concentrações de oxigênio da água, o que proporciona condições desfavoráveis para o estabelecimento de muitos táxons

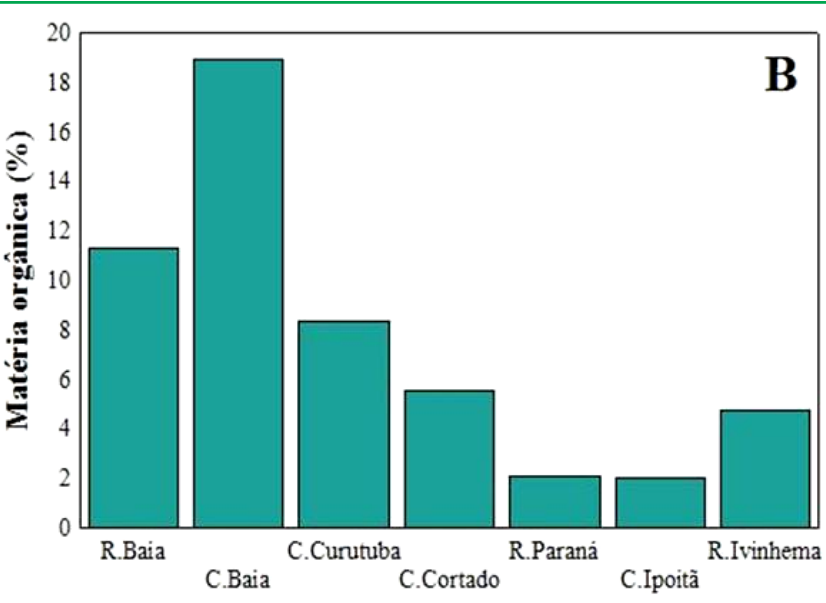

Ambientes

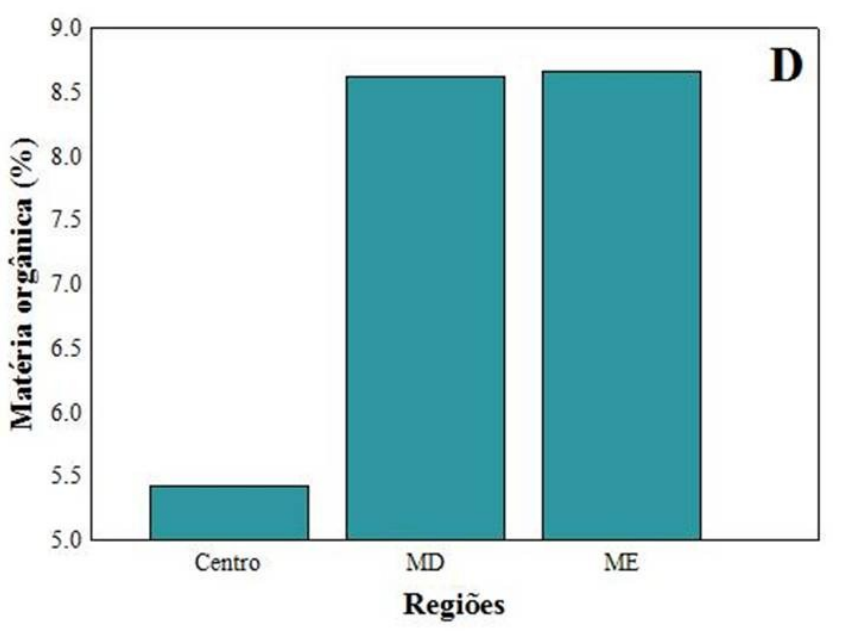

sensíveis a essas condições, como foi verificado por Anjos et al. (2011) para Chironomidae.

O bivalve invasor Limnoperna fortunei (Dunker, 1857) e Chironomidae foram os táxons mais abundantes e de maior ocorrência nas áreas amostradas (Figura 4). $\mathrm{Na}$ região central do canal Curutuba foi registrada alta densidade de L. fortunei associada à predominância de seixos e grânulos, condições estas requeridas por esta espécie para que ocorra sua fixação e estabelecimento no ambiente (MORTON, 1973). Chironomidae foi o grupo mais representativo na maioria dos ambientes analisados, resultado já esperado por ser reconhecido como um dos grupos mais abundantes e diversos na planície de 
TABELA 2: Lista de táxons de invertebrados bentônicos encontrados em rios e canais da planície de inundação do alto rio Paraná no ano de 2010.

\begin{tabular}{|c|c|c|c|c|c|c|c|}
\hline & R. Baía & C. Baía & C. Cortado & C. Curutuba & C. Ipoitã & R. Ivinhema & R. Paraná \\
\hline \multicolumn{8}{|c|}{ PLATYHELMINTHES } \\
\hline Turbellaria & - & - & - & $\mathrm{X}$ & - & $\mathrm{X}$ & - \\
\hline NEMATODA & $\mathrm{X}$ & $\mathrm{X}$ & $\mathrm{X}$ & $\mathrm{X}$ & $\mathrm{X}$ & $\mathrm{X}$ & $\mathrm{X}$ \\
\hline \multicolumn{8}{|l|}{ MOLLUSCA } \\
\hline Bivalvia & $\mathrm{X}$ & $\mathrm{X}$ & $\mathrm{X}$ & $\mathrm{X}$ & $\mathrm{X}$ & $\mathrm{X}$ & $\mathrm{X}$ \\
\hline Limnoperna fortunei & $\mathrm{X}$ & $\mathrm{X}$ & $\mathrm{X}$ & $\mathrm{X}$ & $\mathrm{X}$ & $\mathrm{X}$ & $\mathrm{X}$ \\
\hline Gastropoda & - & $\mathrm{X}$ & $\mathrm{X}$ & $\mathrm{X}$ & $\mathrm{X}$ & $\mathrm{X}$ & $\mathrm{X}$ \\
\hline \multicolumn{8}{|l|}{ ANNELIDA } \\
\hline Hirudinea & $\mathrm{X}$ & $\mathrm{X}$ & - & $\mathrm{X}$ & $\mathrm{X}$ & $\mathrm{X}$ & $\mathrm{X}$ \\
\hline \multicolumn{8}{|l|}{ Oligochaeta } \\
\hline Pristininae & $\mathrm{X}$ & $\mathrm{X}$ & $\mathrm{X}$ & $\mathrm{X}$ & $\mathrm{X}$ & $\mathrm{X}$ & $X$ \\
\hline Naidinae & $\mathrm{X}$ & $\mathrm{X}$ & $\mathrm{X}$ & $\mathrm{X}$ & $\mathrm{X}$ & $\mathrm{X}$ & $\mathrm{X}$ \\
\hline Narapidae & - & - & $\mathrm{X}$ & - & $\mathrm{X}$ & $\mathrm{X}$ & $\mathrm{X}$ \\
\hline Tubificinae & $\mathrm{X}$ & $\mathrm{X}$ & $\mathrm{X}$ & $\mathrm{X}$ & $\mathrm{X}$ & $\mathrm{X}$ & $\mathrm{X}$ \\
\hline Haplotaxidae & - & - & - & - & $\mathrm{X}$ & $\mathrm{X}$ & $X$ \\
\hline \multicolumn{8}{|l|}{ ARTHROPODA } \\
\hline \multicolumn{8}{|l|}{ Arachnida } \\
\hline Acari & $X$ & $X$ & $X$ & $X$ & $X$ & $\mathrm{X}$ & $\mathrm{X}$ \\
\hline \multicolumn{8}{|l|}{ Crustacea } \\
\hline Ostracoda & $X$ & $X$ & $X$ & $X$ & $X$ & $X$ & $\mathrm{X}$ \\
\hline Cyclopoida & $X$ & $X$ & $\mathrm{X}$ & $X$ & $X$ & $X$ & $X$ \\
\hline Calanoida & $X$ & - & $\mathrm{X}$ & $X$ & $X$ & $X$ & $X$ \\
\hline Harpacticoida & - & - & $X$ & - & - & $\mathrm{X}$ & $X$ \\
\hline Amphipoda & - & - & $X$ & - & - & $X$ & - \\
\hline Cladocera & - & $X$ & - & $X$ & $X$ & $X$ & - \\
\hline \multicolumn{8}{|l|}{ Hexapoda } \\
\hline Collembola & - & - & $\mathrm{X}$ & - & $\mathrm{X}$ & $\mathrm{X}$ & $X$ \\
\hline Ephemeroptera & $\mathrm{X}$ & $\mathrm{X}$ & $\mathrm{X}$ & $\mathrm{X}$ & $\mathrm{X}$ & $\mathrm{X}$ & $\mathrm{X}$ \\
\hline Odonata & - & $\mathrm{X}$ & $\mathrm{X}$ & - & - & $\mathrm{X}$ & $\mathrm{X}$ \\
\hline Chironomidae & $\mathrm{X}$ & $\mathrm{X}$ & $\mathrm{X}$ & $\mathrm{X}$ & $\mathrm{X}$ & $\mathrm{X}$ & $\mathrm{X}$ \\
\hline Ceratopogonidae & $\mathrm{X}$ & $X$ & $X$ & $X$ & $X$ & $X$ & $X$ \\
\hline Chaoboridae & $\mathrm{X}$ & - & - & - & $X$ & - & - \\
\hline Trichoptera & $\mathrm{X}$ & $\mathrm{X}$ & - & $X$ & - & $X$ & $X$ \\
\hline Coleoptera & - & - & $X$ & $X$ & $X$ & $X$ & - \\
\hline Lepidoptera & - & $\mathrm{X}$ & - & - & - & - & $X$ \\
\hline Número de táxons & 16 & 18 & 20 & 19 & 21 & 25 & 22 \\
\hline
\end{tabular}


FIGURA 3: Densidade média (ind. $/ \mathrm{m}^{2}$ ) e riqueza total de táxons nos rios e canais amostrados na planície de inundação do alto rio Paraná no ano de 2010. RBai $=$ rio Baía; CCor= canal Cortado; CCur= canal Curutuba; CIpo= canal Ipoitã; RIvi= rio Ivinhema; $\mathbf{R P a r}=$ rio Paraná e CBai $=$ canal Baía.

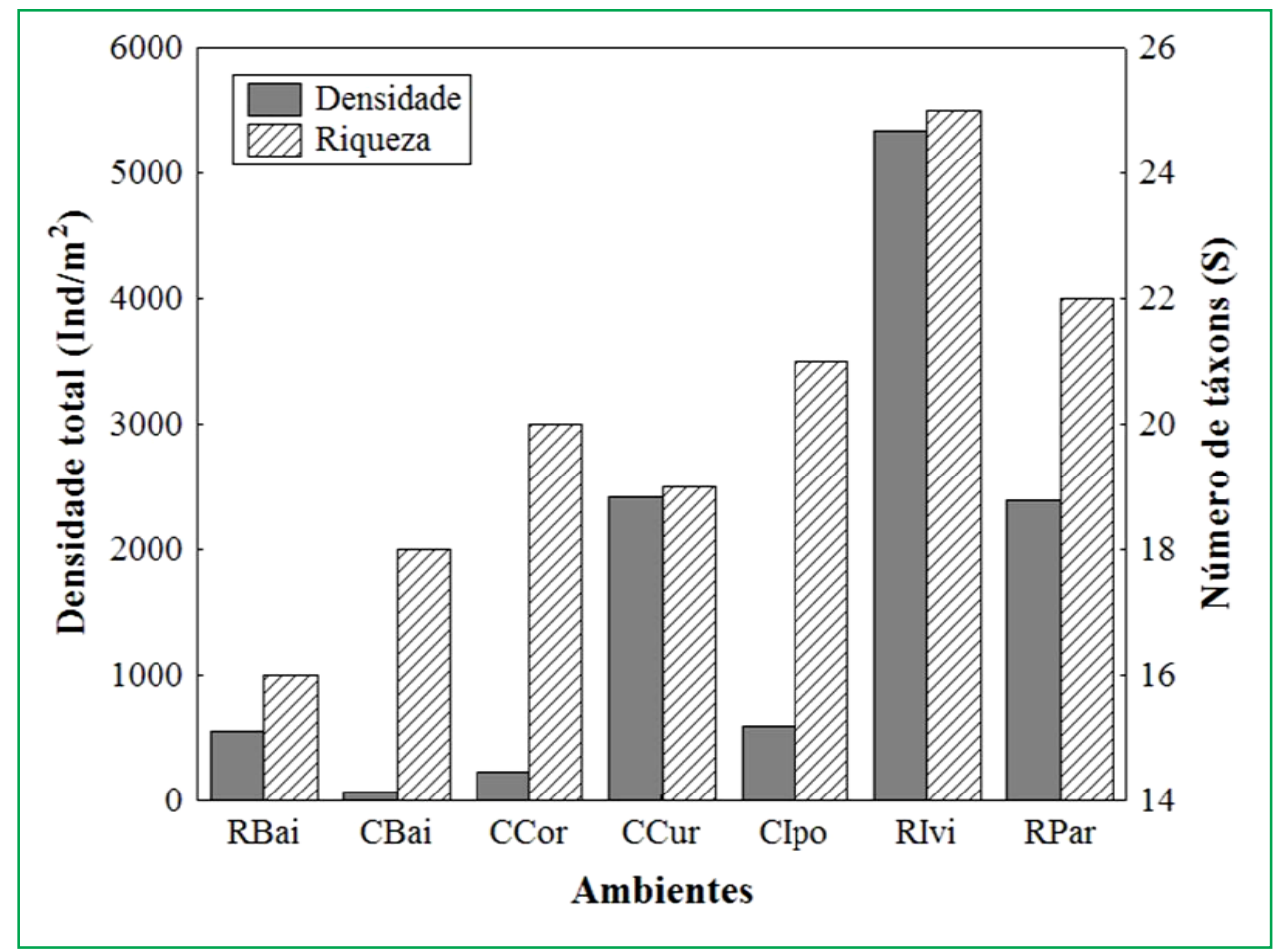

FIGURA 4: Densidade média de invertebrados bentônicos (indivíduos $/ \mathrm{m}^{2}$ ) nas regiões centrais e marginais dos rios e canais da planície de inundação do alto rio Paraná no ano de 2010. $\mathbf{C}=$ região central, $\mathbf{M D}=$ margem direita; $\mathbf{M E}=$ margem esquerda; $\mathbf{R B a i}=$ rio Baía; CCor $=$ canal Cortado; $\mathbf{C C u r}=$ canal Curutuba; $\mathbf{C I p o}=$ canal Ipoitã; $\mathbf{R I v i}=$ rio Ivinhema; $\mathbf{R P a r}=$ rio Paraná e $\mathbf{C B a i}=$ canal Baía.

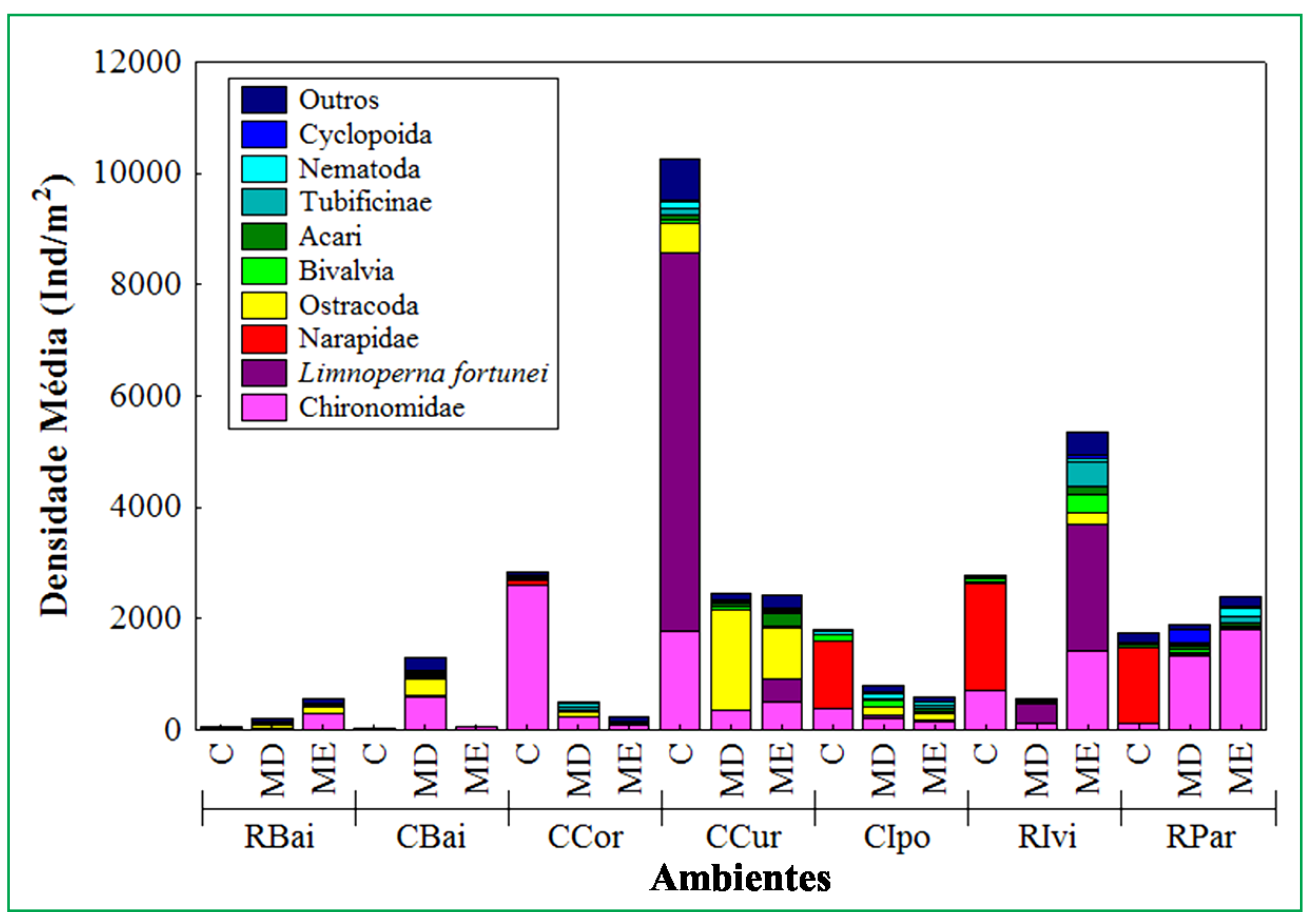


inundação do alto rio Paraná (HIGUTI; TAKEDA, 2002; ROSIN et al., 2009).

Oligochaeta, Chironomidae e Bivalvia foram abundantes e amplamente distribuídos e, devido a pouca mobilidade no sedimento e sua forte associação ao mesmo, podem refletir bem as possíveis alterações ambientais das variáveis físicas e químicas da água e do sedimento (TAKEDA, 1999; GALDEAN et al., 2000; BAPTISTA, 2008), o que os tornam interessantes ferramentas para o biomonitoramento ambiental.
Os dois primeiros eixos da CCA $(\mathrm{p}<0,01)$ explicaram $58,8 \%$ da variabilidade total dos dados utilizados (Figura 5). Os centros dos rios Ivinhema e Paraná e canal Ipoitã foram agrupados (Figura 5C), associados aos altos valores de profundidade, velocidade e areia muito grossa (Figura 5A), e por Collembola, Narapidae, Haplotaxidae e Harpacticoida (Figura 5B). Tais resultados demonstram que esses táxons são adaptados as condições ambientais típicas das regiões centrais de ambientes lóticos. Por outro lado, os canais

FIGURA 5: Diagrama de ordenação para os dois primeiros eixos da Análise de Correspondência Canônica (CCA) em relação aos fatores abióticos (A) e bióticos (B) que influenciaram os eixos na ordenação dos rios e canais da planície de inundação do alto rio Paraná no ano de 2010 (C). Regiões centrais = pontos vazados; regiões marginais $=$ pontos preenchidos. Para os códigos dos pontos: $\mathbf{C}=$ região central; $\mathbf{M}=$ região marginal; $\mathbf{R B a i}=$ rio Baía; $\mathbf{C C o r}=$ canal Cortado; $\mathbf{C C u r}=$ canal Curutuba; $\mathbf{C I p o}=\mathbf{c a n a l}$ Ipoitã; RIvi= rio Ivinhema; $\mathbf{R P a r}=$ rio Paraná e $\mathbf{C B a i}=$ canal Baía.

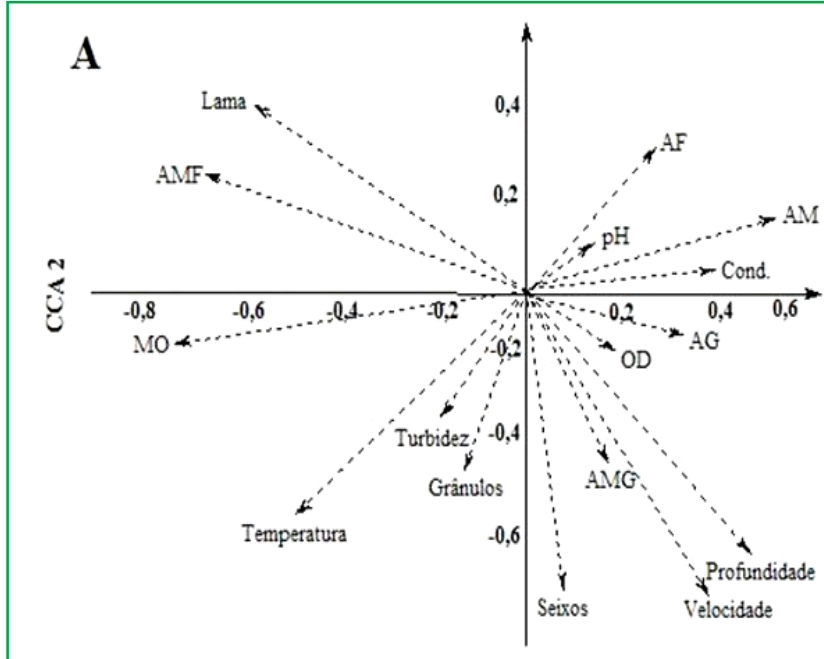

CCA I

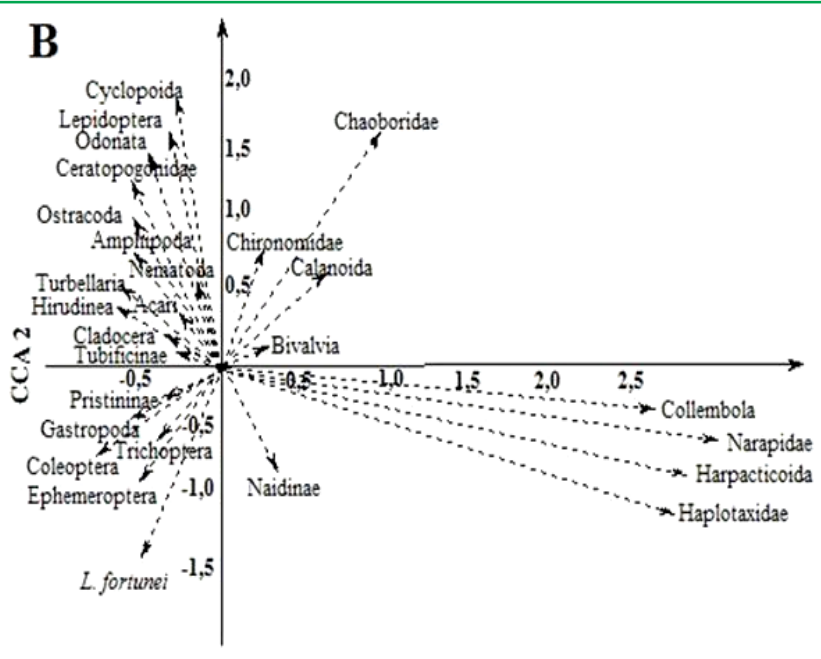

CCA 1

C

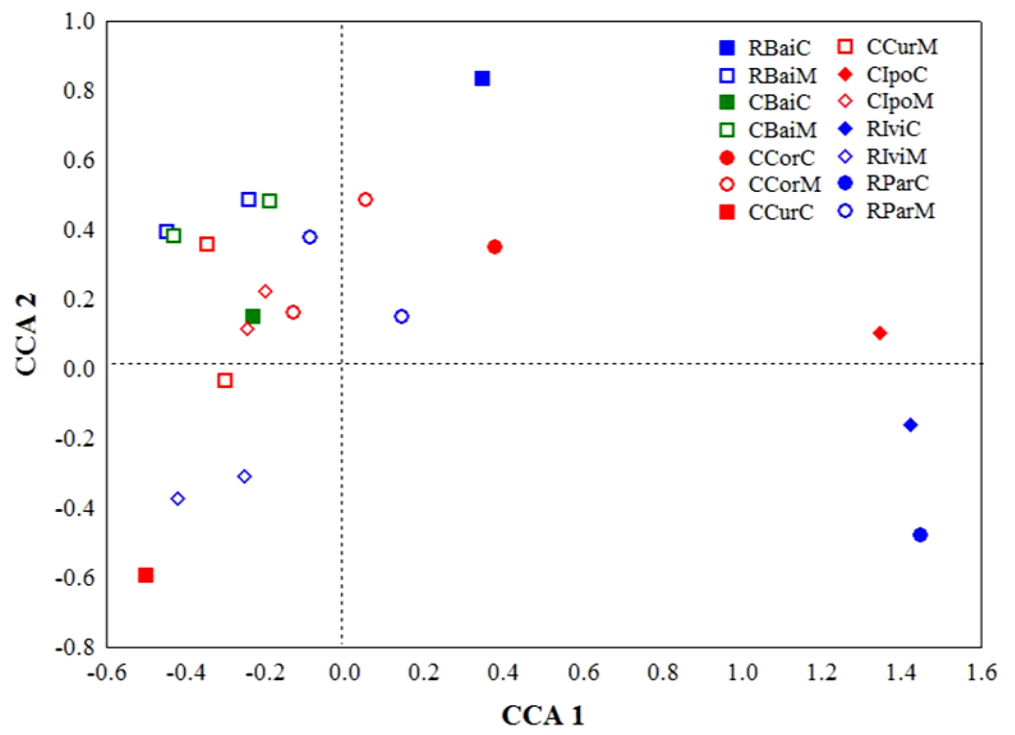


Cortado e Baía, rio Baía e a maioria das margens dos demais ambientes foram agrupados, influenciados principalmente pelos maiores teores de matéria orgânica, lama e areia muito fina, com muitos táxons relacionados a essas características. A alta densidade de L. fortunei agrupou as margens do rio Ivinhema com a região central do canal Curutuba, o que indica que estes locais possuem características ambientais similares, capazes de suportar grandes densidades da espécie.

A maioria dos invertebrados ocorreu nas margens dos canais, exceto Narapidae, Haplotaxidae e Harpacticoida encontrados nas regiões centrais. O tipo de substrato, velocidade de correnteza, concentrações de oxigênio e profundidade são importantes fatores que influenciam a biota aquática em ambientes lóticos (TOWNSEND et al., 1997; BOND; DOWNES, 2000; OLSEN et al., 2001). A CCA mostrou que a maioria dos táxons presentes nas regiões marginais foi influenciada pela matéria orgânica das margens e menor velocidade de fluxo, mostrando que esse tipo de ambiente é favorável à maioria dos invertebrados bentônicos.

Pode-se concluir que a velocidade de fluxo, textura granulométrica e matéria orgânica do sedimento foram fatores estruturadores da comunidade de invertebrados bentônicos, determinando a distribuição dos invertebrados tanto entre os diferentes canais como entre as regiões marginais e centrais desses ambientes, oferecendo maior ou menor disponibilidade de recursos e heterogeneidade ambiental.

\section{Agradecimentos}

Agradecemos o projeto CNPq/PELD/UEM/ Nupelia (Processo 4280/2010) pelo financiamento e ao laboratório de Limnologia Básica do Nupelia da Universidade Estadual de Maringá pelo fornecimento de alguns dos dados dos parâmetros abióticos utilizados.

\section{Referências}

Agostinho, A. A.; ThOMAZ, S. M.; GOMES, L. C. Conservação da biodiversidade em águas continentais do Brasil. Megadiversidade, Belo Horizonte, v. 1, n. 1, p. 70-78, 2005.

ANJOS, A. F.; TAKEDA, A. M.; PINHA, G. D. Distribuição espacial e temporal das larvas de Chironomidae em diferentes ambientes do complexo - rio Baía - Mato Grosso do Sul - Brasil. Acta Scientiarum Biological Sciences, Maringá, v. 33, n. 4, p. 417-426, 2011.

BAPTISTA, D. F. Uso de macroinvertebrados em procedimentos de biomonitoramento em ecossistemas aquáticos. Oecologia Brasiliensis, Rio de Janeiro, v. 12, n. 3, p. 425-441, 2008.

BECHARA, J.A. The relative importance of water quality, sediment composition and floating vegetation in explaining themacrobenthic community structure of floodplain lakes (Paraná River, Argentina). Hydrobiologia, Brussels, v. 333, n. 2, p. 95-109, 1996.

BERGAMIN, H.; REIS, B. F.; ZAGATTO, E. A. G., A new device for improving sensitivity and stabilization in flow injection analysis. Analytica Chimica Acta, Amsterdam, v. 97, p. 427-431, 1978.

BOND, N. R.; DOWNES, B. J. Flow-related disturbance in streams: an experimental test of the role of rock movement in reducing macroinvertebrates population densities. Marine and Freshwater Research, Collingwood, v. 51, p. 333-337, 2000.

BUCKUP, L.; BUENO, A. A. P.; BOND-BUCKUP, G.; CASAGRANDE, M.; MAJOLO, F. The benthic macroinvertebrate fauna of highland streams in southern Brazil: composition, diversity and structure. Revista Brasileira de Zoologia, Curitiba, v. 24, n. 2, p. 294-301, 2007.

BUSS, D. F.; BAPTISTA, D. F.; NESSIMIAN, J. L.; EGLER, M. Substrate specificity, environmental degradation and disturbance structuring macroinvertebrate assemblages in neotropical streams. Hydrobiologia, Brussels, v. 518, n. 1-3, p. 179-188, 2004.

CALLISTO, M.; MORENO, P.; BARBOSA, F. A. R. Habitat diversity and benthic functional trophic groups at Serra do Cipó, southeast Brazil. Brazilian Journal of Biology, São Carlos, v. 61, n. 2, p. 259-266, 2001

GALDEAN, N.; CALliStO, M.; BARBOSA, F. A. R. Lotic ecosystems of Serra do Cipó, southeast Brazil: water quality and a tentative classifcation based on the benthic macroinvertebrate community. Aquatic Ecosystem Health and Management, Ontario, v. 3, p. 545-552, 2000.

GINÉ, M. F.; BERGAMIN, F. H.; ZAGATTO, E. A. G.; REIS, B. F. Simultaneous determination of nitrate and nitrite by flow injection analysis. Analytica Chimica Acta, Amsterdam, v. 114, p. 191-197, 1980.

GOLTERMAN, H. L.; ClYMO, R. S.; OHMSTAD, M. A. M. Methods for physical and chemical analysis of fresh water. Oxford: Blackwell Scientific, 1978. 214 p.

HENRIQUES-OLIVEIRA, A. L.; SANSEVERINO, A. M.; NESSIMIAN, J. L. Larvas de Chironomidae (Insecta: Diptera) de substrato rochoso em dois rios em diferentes estados de preservação na Mata Atlântica, RJ. Acta Limnologica Brasiliensia, Botucatu, v. 11, n. 2, p. 17-28, 1999.

HENRIQUES-OLIVEIRA, A. L.; DORIVILLÉ, L. F. M.; NESSIMIAN, J. L. Distribuition of Chironomidae larvae fauna (Insecta: Diptera) on different substrates in stream at Floresta da Tijuda, RJ, Brasil. Acta Limnologia Brasiliensia, Botucatu, v. 15, n. 2, p. 69-84, 2003.

HIGUTI, J.; TAKEDA, A. M. Spatial and temporal variation in of Chironomid larval (Diptera) in two lagoons and two tributaries of the Upper Paraná River floodplain, Brazil. Brazilian Journal of Biology, São Carlos, v. 62, n. 4, p. 807-818, 2002. 
KOWNACKI, A.; GALAS, J.; DUMINICKA, E.; MIELEWCZYK, $\mathrm{S}$. Invertebrate communities in permanent and temporary high mountain lakes (Tatra Mts). Annales de Limnologie, Santa Fé, v. 36, n. 3, p.181-188, 2000.

LAKE, P. S. Disturbing hard and soft bottom communities: a comparison of marine and freshwater environments. Australian Journal of Ecology, Carlton, v. 15, p. 477-488, 1990.

LEGENDRE, P.; LEGENDRE, L. Numerical ecology. Amsterdam: Elsevier, 1998. 853 p.

MacKERETH, F. Y. H.; HERON, J.; TALLING, J. J. Water analysis: some revised methods for Limnologists. Freshwater Biological Association, Cumbria, v. 36, p. 1-120, 1978.

MARQUES, M. G. S. M.; FERREIRA, R. L; BARBOSA, F. A. $R$. A comunidade de macroinvertebrados aquáticos e características limnológicas das Lagoas Carioca e da Barra, Parque Estadual do Rio Doce, MG. Brazilian Journal of Biology, São Carlos, v. 59, n. 2, p. 203-210, 1999.

MONTANHOLI-MARTINS, M. C.; TAKEDA, A. M. Communities of benthic Oligochaetes in relation to sediment structure in the upper Paraná River, Brazil. Studies on Neotropical Fauna and Environment, Tübingen, v. 34, p. 52-58, 1999.

MOORE, J. E.; SWIHART, R. K. Toward ecologically explicit null models of nestedness. Oecologia, Heidelberg, v. 152, p. 763-777, 2007.

MORETTI, M. S.; CALLISTO, M. Biomonitoring of benthic macroinvertebrates in the middle Doce River watershed. Acta Limnologica Brasiliensia, Botucatu, v. 17, p. 267-282, 2005.

MORTON, B. Some aspects of the biology and functional morphology of the organisms, of feeding and digestion of Limnoperna fortunei (Dunker) (Bivalvia: Mytilidae). Malacologia, Ann Arbor, Michigan, v. 12, n. 2, p. 265-281, 1973.

MUGNAI, R.; NESSIMIAN, J. L.; BAPTISTA, D. L. Manual de identificação de macroinvertebrados aquáticos do Estado do Rio de Janeiro. Rio de Janeiro: Technical Books, 2010. 173 p.

OLSEN, D. A.; TOWNSEND, C. R.; MATTHAEI, C. D. Influence of reach geomorphology on hyporheic communities in a gravelbed stream. New Zealand Journal of Marine \& Freshwater Research, Wellington, v. 35, p. 181-190, 2001.

PAILlEX, A.; CASTEllA, E.; CARRON, G. Aquatic macroinvertebrate response along a gradient of lateral connectivity in river floodplain channels. Journal of the North American Benthological Society, Lawrence, v. 26, n. 4, p. 779-796, 2007.

PÉREZ, G. R. Guía para el estúdio de los macroinvertebrados acuáticos del Departamento de Antioquia. Bogotá: Editorial Presencia Ltda., 1988. 217 p.

POFF, N. L. Landscape filters and species traits: towards mechanistic understanding and prediction in stream ecology. Journal of the North American Benthological Society, Lawrence, v. 16, p. 391409, 1997.

PRÍNCIPE, R. E.; CORIGLIANO, M. C. Benthic, drifting and marginal macroinvertebrate assemblages in a lowland river: temporal and spatial variations and size structure. Hydrobiologia, Brussels, v. 553, p. 303-317, 2006.

RIBEIRO, L. O.; UIEDA, V. S. Estrutura da comunidade de macroinvertebrados bentônicos de um riacho de serra em Itatinga, São Paulo, Brasil. Revista Brasileira de Zoologia, Curitiba, v. 22, n. 3, p. 613-618, 2005.
RICKLEFS, R. E. Community diversity: relative roles of local and regional processes. Science, New York, v. 235, p. 167-171, 1987.

ROSIN, G. C.; OLIVEIRA-MANGAROTTI, D. P.; TAKEDA, A. M. Chironomidae (Diptera) community structure in two subsystems with different states of conservation in a floodplain of southern Brazil. Acta Limnologica Brasiliensia, Botucatu, v. 22, n. 3, p. 276-286, 2010.

ROSIN, G. C.; OLIVEIRA-MANGAROTTI, D. P.; TAKEDA, A. M.; BUTAKKA, C. M. M. Consequences of a dam construction upstream from the Upper Paraná River floodplain (Brazil): temporal analysis of the Chironomidae community over an eight-year period. Brazilian Journal of Biology, São Carlos, v. 69, p. 591-608, 2009.

SILVA, F. L.; MOREIRA, D. C.; RUIZ, S. S.; BOCHINI, G. L. Avaliação da importância da unidade de conservação na preservação d diversidade de Chironomidae (Insecta: Diptera) no córrego Vargem Limpa, Bauru, Estado de São Paulo. Acta Scienciarum Biological Science, Maringá, v. 29, n. 4, p. 401-405, 2007.

SOUZA FILHO, E. E.; STEVAUX, J. C. Geologia e Geomorfologia do complexo rio Baía, Curutuba, Ivinhema. In: VAZZOLER, A. E. A. M.; AGOSTINHO, A. A; HAHN, N. S. (Ed.). A planície de inundação do alto rio Paraná. Maringá: EDUEM, 1997. 460 p.

SÚAREZ, Y. R.; PETRERE, J. R. M.; CATELLA, A. C. Factors determining the structure of fish communities in Pantanal lagoons (MS, Brazil). Fisheries Management and Ecology, East Yorkshire, v. 8, p. 173-186, 2001.

SURIANO, M. T.; FONSECA-GESSNER, A. A. Chironomidae (Diptera) larvae in stream of Parque Estadual de Campos do Jordão, São Paulo State, Brasil. Acta Limnologica Brasiliensia, Botucatu, v. 16, n. 2, p. 126-136, 2004.

TAKEDA, A. M. Oligochaeta community of alluvial upper Paraná River, Brazil: spatial and temporal distribution (1987-1988). Hydrobiologia, Brussels, v. 412, p. 35-42, 1999.

TAKEDA, A. M.; FUJITA, D. S. Benthic invertebrates. In: THOMAZ S. M.; AGOSTINHO A. A.; HAHS N. S. (Ed.). The Upper Paraná River and its floodplain: physical aspects, ecology and conservation. Leiden: Backhuys Publishers, 2004. p. 75-102.

TAKEDA, A. M.; KOBAYASHI, J. T.; RESENDE, D. L. M. C.; FUJITA, D. S.; AVELINO, G. S.; FUJITA, R. H.; PAVAN, C. B.; BUTAKKA, C. M. M. Influence of decreased water level on the Chironomidae community of the Upper Paraná River alluvial plain. In: AGOSTINHO, A. A.; RODRIGUES, L.; GOMES, L. C.; THOMAZ, S. M.; MIRANDA, L. E. (Ed.). Structure and functioning of the Paraná River and its floodplain. Maringá: Eduem, 2004. p. 101-106.

TER BRAAK, C. J. F. Canonical correspondence analysis: a new eigenvector technique for multivariate direct gradient analysis. Ecology, New York, v. 67, p. 1167-1179, 1986.

THOMAZ, S. M.; BINI, L. M.; BOZELLI, R. L. Floods increase similarity among aquatic habitats in river-floodplains systems. Hydrobiologia, Brussels, v. 579, n. 1, p. 1-13, 2007.

TOWNSEND, C. R.; ABRUCKLE, C. J.; CROWL, T. A.; SCARSBROOK, M. R. The relationship between land use and physicochemistry, food resources and macroinvertebrate communities in tributaries of the Taieri River, New Zealand: a hierarchically scaled approach. Freshwater Biology, London, v. 37, p. 177-191, 1997. 
UNIVERSIDADE ESTADUAL DE MARINGÁ. A planície de inundação do alto rio Paraná: Site $6 \mathrm{PELD} / \mathrm{CNPq}$ - Relatório anual (2000). AGOSTINHO, A. A.; THOMAZ, S. M.; NAKATANI, K. (Coord.). Maringá: Nupelia/PELD/CNPq, 2000, p. 123-129. Acesso em: http://www.peld.uem.br/Relat2000.

WENTWORTH, C. K. A escale of grade and class terms for clastic sediments. The Journal of Geology, Chicago, v. 30, p. 377-392, 1922.
WÜRDIG, N. L.; CENZANO, C. S. S.; MOTTA-MARQUES, D. Macroinvertebrate communities structure in different environments of the Taim Hydrological System in the state of Rio Grande do Sul, Brazil. Acta Limnologica Brasiliensis, Botucatu, v. 19, n. 4, p. 427-438, 2007. 